TITLE:

\title{
Multiaffine chemical turbulence
}

$\operatorname{AUTHOR}(\mathrm{S})$ :

Kuramoto, Y; Battogtokh, D; Nakao, H

CITATION:

Kuramoto, Y ... [et al]. Multiaffine chemical turbulence. PHYSICAL REVIEW LETTERS 1998, 81(16): 3543-3546

ISSUE DATE:

1998-10-19

URL:

http://hdl.handle.net/2433/50552

RIGHT:

Copyright 1998 American Physical Society 


\title{
Multiaffine Chemical Turbulence
}

\author{
Yoshiki Kuramoto, Dorjsuren Battogtokh,* and Hiroya Nakao \\ Department of Physics, Graduate School of Sciences, Kyoto University, Kyoto 606-8502, Japan
}

(Received 2 June 1998)

\begin{abstract}
A three-component reaction-diffusion model is proposed as the first example to exhibit chemical turbulence with multiaffine fractal structures, the underlying mechanism being the same as for similar turbulence discovered recently in some nonlocally coupled oscillator systems. The role played by the strongly diffusive component can be substituted by a long-wave random force, and this idea leads to our proposal of the second, far simpler reaction-diffusion model given by the randomly driven FitzHughNagumo nerve conduction equation. [S0031-9007(98)07376-1]
\end{abstract}

PACS numbers: 82.20.Fd, 05.45.+b, 47.27. $-\mathrm{i}, 47.53 .+\mathrm{n}$

Spontaneous spatiotemporal disorder through chemical reaction and diffusion is called chemical turbulence around which considerable amounts of work have been done over the last two decades [1,2]. Some possible forms of chemical turbulence have been proposed, though without clear evidence for their existence in real chemical reactions.

The goal of this paper is to propose yet another form of chemical turbulence which, besides being conceptually new, could be much easier to realize experimentally. The main characteristic of our chemical turbulence is the spatial multiscaling and intermittency reminiscent of fully developed fluid turbulence [3]. We found that it belongs to the same class of spatiotemporal chaos that was discovered recently in various models of nonlocally coupled elements and also in arrays of chaotic maps subjected to long-wave random forces [4-6]. The discovery of the same behavior in reaction-diffusion systems, i.e., a representative class of nonlinear dissipative media with local coupling, will therefore strengthen the indication that the related phenomena are so universal in the real world.

We shall propose two illustrative reaction-diffusion models. The first is a three-component oscillatory system near the onset of oscillation. Our model is particularly instructive in that it reveals a limitation of the complex Ginzburg-Landau (CGL) equation in its application to self-oscillatory media even for the purpose of a most qualitative understanding. The second model, which will be discussed only briefly, is given by a nonautonomous two-component system of the FitzHugh-Nagumo type [7]. This model seems more relevant to experiment, because we learn from it a practical advantage of working with forced reaction-diffusion systems. With such systems, one may in fact circumvent the most difficult problem of producing intrinsic spatiotemporal chaos; singular turbulent fluctuations are generated from long-wave nonsingular fluctuations fed into the system by random forcing.

We will not attempt detailed analysis of the multiaffine/ multifractal properties themselves. This could be done much more efficiently with the use of arrays of chaotic maps.

Consider a reaction-diffusion system in one spatial dimension:

$$
\begin{aligned}
\frac{\partial X}{\partial t} & =A-(B+1) X+X^{2} Y+K_{X} S, \\
\frac{\partial Y}{\partial t} & =B X-X^{2} Y+K_{Y} S, \\
\epsilon \frac{\partial S}{\partial t} & =-S+D \frac{\partial^{2} S}{\partial x^{2}}+X
\end{aligned}
$$

where $A, B, K_{X}, K_{Y}, D$, and $\epsilon$ are constants. Under the condition $B>1+A^{2}$, the set of the first two equations with $S=0$ represents a limit-cycle oscillator called the Brusselator [8]. Thus, the above system may be viewed as a large population of oscillators interacting indirectly via an inactive diffusive component. Similar situations may be met in a variety of cellular assemblies in living systems [9], where the cell activity, assumed timeperiodic, is controlled by the local concentration of some diffusive chemical substance which in turn is produced from each cell with the rate of production depending on the cell activity. It was speculated earlier [4] that reaction-diffusion systems of this class might give rise to turbulence with spatial multiscaling. Equations (1) indeed provide the first example justifying this anticipation.

Our numerical analysis of (1) was carried out on a system of unit length satisfying periodic boundary conditions. The uniqueness of the turbulence discovered may be seen from Fig. 1 where space-time patterns of $X$ and $S$ are illustrated in the gray scale. While the spatial smoothness of $S$ could be understood from its diffusive nature, the highly intermittent pattern of $X$ is remarkable and needs to be explained. The strong resemblance of the latter with some patterns obtained in nonlocally coupled systems [4] implies the equivalence in the nature of turbulence between these two classes of systems. In particular, we expect that the patterns of $X$ (and also $Y$ ) at given $t$ are characterized, as far as their spatial continuity is preserved, by multiaffine fractals [10] over some range of length scale up to the diffusion length $\sqrt{D}$ of $S$. This means that various moments of the amplitude increment $y \equiv\left|X\left(x_{0}+x\right)-X\left(x_{0}\right)\right|$ over a sufficiently short distance $x$ obey a power law $\left\langle y^{q}\right\rangle \sim x^{\alpha(q)}$ ( $q>0$ ) with the exponent $\alpha(q)$ having nonlinear dependence on $q$. A theory developed previously [5,6], based 


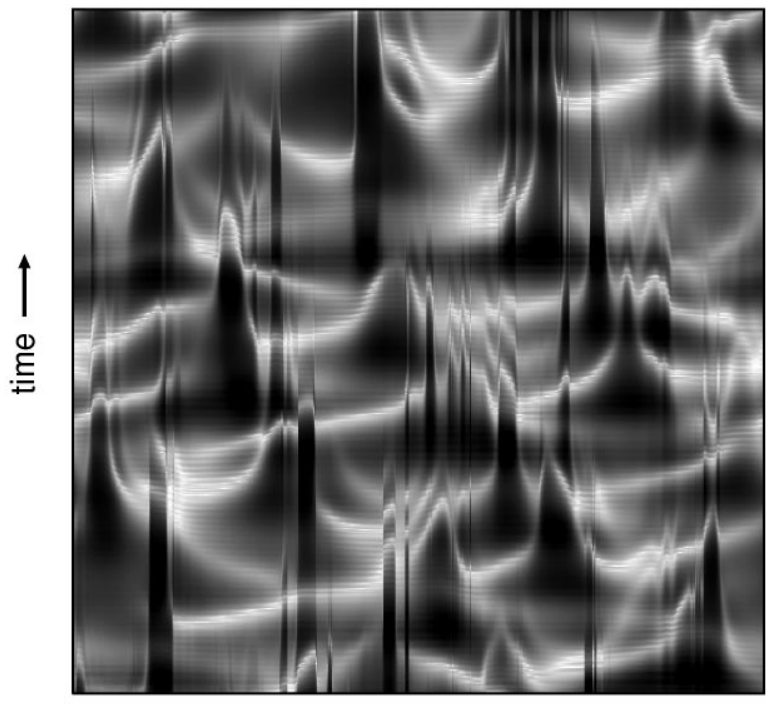

space

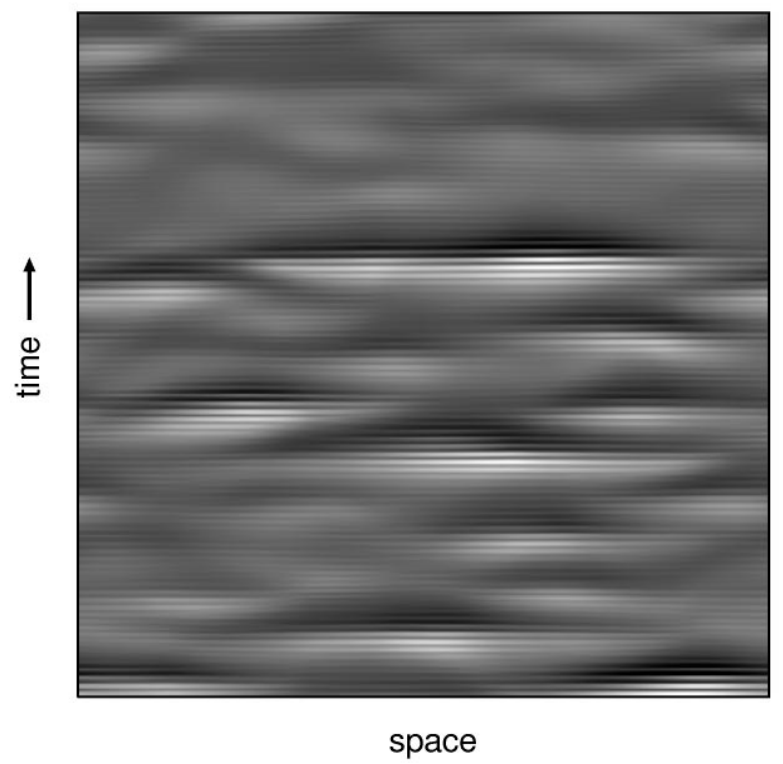

FIG. 1. Spatiotemporal patterns of $X$ (top) and $S$ (bottom) in gray scale, where the darker (lighter) regions correspond to larger (smaller) $X$ or $S$. The space and time spans are 1.0 and 327.7 , respectively. The patterns were obtained after a temporal coarse graining of the time sequences at each $x$ over the period $\Delta t=3.2$, the average period of the individual oscillation. Parameter values are $A=1.8, B=6.5, K_{X}=$ $0.28, K_{Y}=-0.058, D=0.02$, and $\epsilon=0.01$.

on the assumption of a multiplicative stochastic process obeyed by $y$, predicts a simple multiscaling

$$
\alpha(q)=\left\{\begin{array}{ll}
q & (q \leq \beta) \\
\beta & (q>\beta)
\end{array},\right.
$$

where $\beta$ is a parameter-dependent positive constant.

As a quantity which contains a little more information than $\left\langle y^{q}\right\rangle$, we introduce the probability distribution $P(\chi)$ of coarse-grained spatial derivative $\chi \equiv y / x$. Figure 2 shows $P(\chi)$ obtained numerically from (1) for three different levels of coarsening; i.e., $x=2^{-8}, 2^{-11}$, and $2^{-14}$.

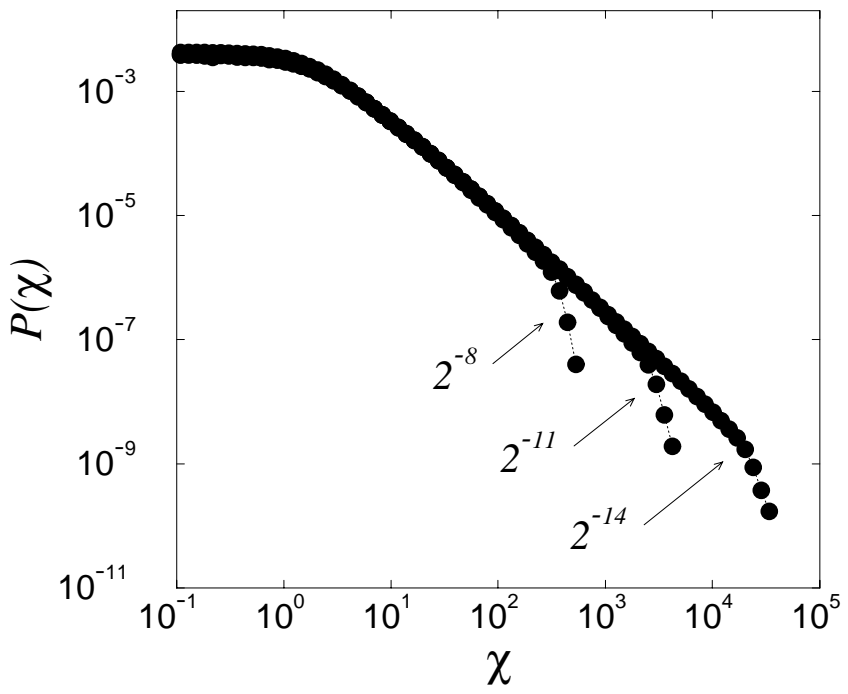

FIG. 2. Log-log plot of the probability densities $P(\chi)$ of the coarse-grained spatial derivative $\chi \equiv y / x$ obtained for different $x$ indicated in the figure. Parameter values are the same as in Fig. 1. Estimated value of $\beta$ is 0.55 .

We first check the consistency of this result with the formula in (2). It is clear from the figure that $P(\chi)$ obeys a power law $P(\chi) \sim \chi^{-(1+\beta)}$ for intermediate values of $\chi$; its power-law tail has a cutoff at about $\chi \sim x^{-1}$, while its power-law divergence with decreasing $\chi$ starts to be suppressed around some value independent of $x$. Consequently, the probability distribution $Q(y) \equiv P(y / x) x^{-1}$ of $y$ should obey the same power law for intermediate $y$, but showing a sudden drop near some $y$ independent of $x$ and saturating below $y \sim x$. Such behavior of $Q(y)$ may be cast into a simple model $Q(y)=$ const $(y<x)$, $\propto \chi^{-(1+\beta)}(x \leq y<1)$, and $0(y \geq 1)$, thus yielding analytic expressions for $\left\langle y^{q}\right\rangle$ with the exponents identical to the formula in (2) [6].

The first moment $\langle y\rangle$ or the corresponding exponent $\alpha(1)$ is of particular interest for its relation to the fractal dimension of the pattern. In fact, the dimension $D_{f}$ of the $X$ versus $x$ curve is defined by $x^{-1}\langle y\rangle \sim x^{1-D_{f}}$, which implies the relation $D_{f}=2-\alpha(1)$. Via (2), the last equality means $D_{f}=2-\beta$ provided $\beta<1$. Thus, the pattern is fractal if $0<\beta<1$. It is interesting to note that, in contrast to the usual fractal curves, the spatial derivative $d X / d x$ or its absolute value $|d X / d x| \equiv$ $\lim _{x \rightarrow 0} \chi$ seems generally to exist, because from the property of $P(\chi)$ described above the limiting distribution $\lim _{x \rightarrow 0} P(\chi)$ should exist and be normalizable. Nevertheless, the expectation $\langle\chi\rangle$ in the same limit diverges if $\beta<1$, and the last inequality is precisely the condition for the fractality of the pattern.

$Q(y)$ may have a negative exponent $\beta$. Then $\lim _{x \rightarrow 0}\left\langle y^{q}\right\rangle$ will not vanish, implying that the pattern is discontinuous everywhere.

The above numerical analysis of the modified Brusselator (1) was carried out for small $\epsilon$ and near the onset of uniform oscillation, i.e., the situation where the reduction of (1) to the complex Ginzburg-Landau equation may be 
possible [1]. Under the parameter conditions described below, however, this naive reduction idea breaks down due to the appearance of another small parameter comparable with the bifurcation parameter, leading to completely different behavior from what is expected from CGL.

To give a more detailed account, we simplify the problem by considering the limit of small $\epsilon$ and infinite system size. This enables one to eliminate $S$ from (1) adiabatically and obtain a nonlocally coupled two-component system of the form

$$
\begin{aligned}
\frac{\partial X}{\partial t}= & A-\left(B+1-K_{X}\right) X+X^{2} Y \\
& +K_{X} \int_{-\infty}^{\infty} \sigma\left(x-x^{\prime}\right)\left[X\left(x^{\prime}\right)-X(x)\right] d x^{\prime}, \\
\frac{\partial Y}{\partial t}= & \left(B+K_{Y}\right) X-X^{2} Y \\
& +K_{Y} \int_{-\infty}^{\infty} \sigma\left(x-x^{\prime}\right)\left[X\left(x^{\prime}\right)-X(x)\right] d x^{\prime},
\end{aligned}
$$

where $\sigma(x)=\frac{1}{2 \sqrt{D}} \exp (-|x| / \sqrt{D})$. Define new parameters $a=A /\left(1-K_{X}-K_{Y}\right)^{3 / 2}$ and $b=\left(B+K_{Y}\right) /(1-$ $\left.K_{X}-K_{Y}\right)$, where $K_{X}+K_{Y}<1$ is assumed. The critical condition for the onset of oscillation is then given by $b=b_{c}$, where $b_{c}=1+a^{2}$. The standard reduction technique [1] is applied to (3) near $b_{c}$, leading to a CGLtype equation with nonlocal coupling. In terms of a suitably defined complex amplitude $W$ and scaled time $t^{\prime}$, this is expressed as

$$
\begin{aligned}
\frac{\partial W}{\partial t^{\prime}}= & W-\left(1+i c_{2}\right)|W|^{2} W \\
& +k\left(1+i c_{1}\right) \int_{-\infty}^{\infty} \sigma\left(x-x^{\prime}\right) \\
& \times\left[W\left(x^{\prime}\right)-W(x)\right] d x^{\prime},
\end{aligned}
$$

where $k=K_{X} /\left[\mu b_{c}\left(1-K_{X}-K_{Y}\right)\right], \quad c_{1}=-a(1+$ $\left.K_{X}^{-1} K_{Y}\right), c_{2}=\left(4-7 a^{2}+4 a^{4}\right) /\left[3 a\left(2+a^{2}\right)\right]$, and $\mu=$ $\left(b-b_{c}\right) / b_{c}$. Note, however, that the bifurcation parameter $\mu$ has not been scaled out from (4) due to the $\mu$ dependence of $k$. We usually recover the scale invariance of the equation by assuming long-waved $W$ such that the integral may give a quantity of $O(\mu)$. Then the local-coupling approximation (LCA) is allowed, and after the rescaling $x \rightarrow x / \sqrt{\mu}$ (4) is reduced to the usual CGL. In the present case, in contrast, the scale invariance holds simply because $K_{X}=O(\mu)$ or $k=O(1)$ so that LCA is generally not allowed. Actually, the parameter values used in our simulation of (1) give $\mu=0.0464, k=0.988, c_{1}=$ -2.09 , and $c_{2}=2.09$, Moreover, we are rather deep in the Benjamin-Feir unstable regime $1+c_{1} c_{2}<0$ $[1,2]$ where the difference in behavior between the local and nonlocal CGLs becomes decisive. The last three parameter values in fact fall into the domain where (4) was found to exhibit turbulence with nontrivial scaling properties [4,5].
Coming back to the original Eqs. (1), it may also be wondered whether the smallness of $\epsilon$ is crucial to the occurrence of the type of turbulence concerned. Detailed arguments will be developed elsewhere, but we simply show here $\left\langle y^{2}\right\rangle$ obtained numerically for some different $\epsilon$ (Fig. 3). When $\epsilon$ is increased, no qualitative change in the power-law behavior can be seen up to $\epsilon=0.2$, while its sudden breakdown seems to occur somewhere between $\epsilon=0.2$ and 0.3 , the reason for which is yet to be explained.

The final remark on our first model concerns the numerical scheme employed. In contrast to the types of reaction-diffusion dynamics concerned in most of the foregoing studies, the unique feature of our chemical turbulence is the emergence of complicated fine structures at scales much shorter than the diffusion length. Therefore, the usual numerical scheme based on space discretization will not work. Given a set of data of $X(x, t), Y(x, t)$, and $S(x, t)$, our method proceeds as follows. Taking advantage of the linearity of the equation for $S$, we work with the solution of that equation in (1) in the wave number space; i.e.,

$$
\begin{aligned}
S_{k}(t+\Delta t)= & S_{k}(t) \exp \left(-\gamma_{k} \Delta t\right) \\
+\epsilon^{-1} \int_{t}^{t+\Delta t} & \exp \left[-\gamma_{k}(t+\Delta t-\tau)\right] \\
& \times X_{k}(\tau) d \tau,
\end{aligned}
$$

where $S_{k}$ and $X_{k}$ are the spatial Fourier transforms of $S(x)$ and $X(x)$, respectively, and $\gamma_{k}=\epsilon^{-1}\left(1+4 \pi^{2} D k^{2}\right)$. Calculating $X_{k}(\tau)$ approximately from the data of $X(x, t)$ and $\dot{X}(x, t)$, we thus obtain $S_{k}(t+\Delta t)$ and, hence, $S(x, t+\Delta t)$. On the other hand, we work with the $x$ space when dealing with the first and second equations of (1); numerical integration of these ordinary differential equations with the use of the second order Euler scheme

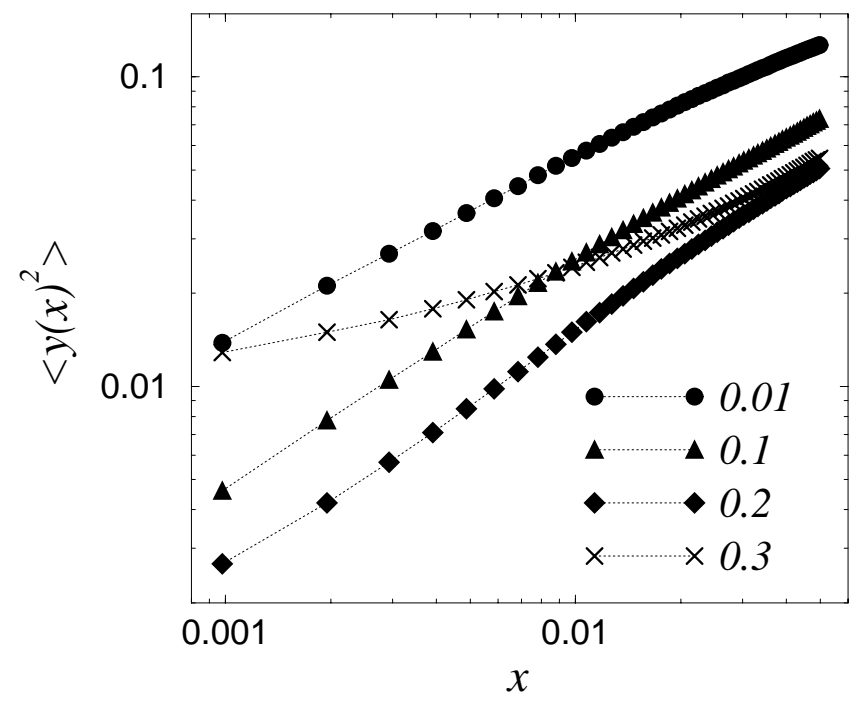

FIG. 3. Log-log plot of the second moments $\left\langle y(x)^{2}\right\rangle$ vs $x$ for some values of $\epsilon$ indicated in the figure. The values of the other parameters are the same as in Fig. 1. 
gives $X(x, t+\Delta t)$ and $Y(x, t+\Delta t)$ to the same approximation as for $S(x, t+\Delta t)$. Note that our method is also not hampered by the smallness of $\epsilon$.

In the model discussed above, $S$ acts similar to a longwave stochastic field driving the individual oscillators which are themselves uncoupled with each other. The dynamics of $S$ and that of the individual elements must be self-consistently interrelated. For certain theoretical as well as empirical reasons [5,6], however, such selfconsistency is not important to the occurrence of the type of turbulence in question. One may then expect similar turbulent behavior simply by replacing $S$ with some space-time dependent field of purely external origin. Experimentally, this fact seems particularly important because applying external random forces would be much easier in practice than finding out systems with intrinsic spatiotemporal chaos. We are thus led to the study of the following nonautonomous system which is the FitzHugh-Nagumo nerve conduction equation under long-wave stochastic forcing:

$$
\begin{aligned}
\frac{\partial U}{\partial t}= & \epsilon^{-1}\left(U-U^{3}-V\right)+D \frac{\partial^{2} U}{\partial x^{2}} \\
& +K \sin [\omega t+x+\psi(t)], \\
\frac{\partial V}{\partial t}= & a U+b .
\end{aligned}
$$

Our choice of the FitzHugh-Nagumo system rather than the Brusselator is due to its stronger similarity to the BelousovZhabotinsky reaction system. In the first equation in (6), the forcing is given by a simple sine wave, while its temporal variation is random through the phase $\psi$ assumed to obey the Langevin equation

$$
\frac{d \psi}{d t}=v, \quad \frac{d v}{d t}=-\gamma v+f(t) .
$$

Here $f(t)$ are the uniform random numbers over an interval $\left[f_{0},-f_{0}\right]$ and chosen independently at each time step. Thus, $\psi$ behaves similar to the position of a Brownian particle on a line, ensuring at the same time the statistical uniformity over the entire system. The system size is fixed at $2 \pi$ and periodic boundary conditions are assumed. If $D=0$, there is no spatial coupling, and this particular case corresponds to our first model where direct diffusive coupling was absent among the constituent oscillators. Figure 4 shows the second moments $\left\langle y^{2}\right\rangle$ of the increment $y \equiv\left|U\left(x_{0}+x\right)-U\left(x_{0}\right)\right|$ as a function of $x$ in the presence and absence of diffusion. The numerical simulation was carried out with parameter values for which the individual elements are nonoscillatory but excitable. We confirmed that, as far as the statistics of the turbulent fluctuations are concerned, there is no qualitative difference between the cases with and without spontaneous oscillation. As expected, in the absence of diffusion, $\left\langle y^{2}\right\rangle$ obeys a power law with nontrivial exponent $\alpha(2) \simeq 0.84$. This is compared in the same figure with the case of nonvanishing $D$ for which the power law is valid only for $x$ sufficiently larger than the diffusion length $\sqrt{\epsilon D}$. Note that the powerlaw regime is also limited from the above by the wave-

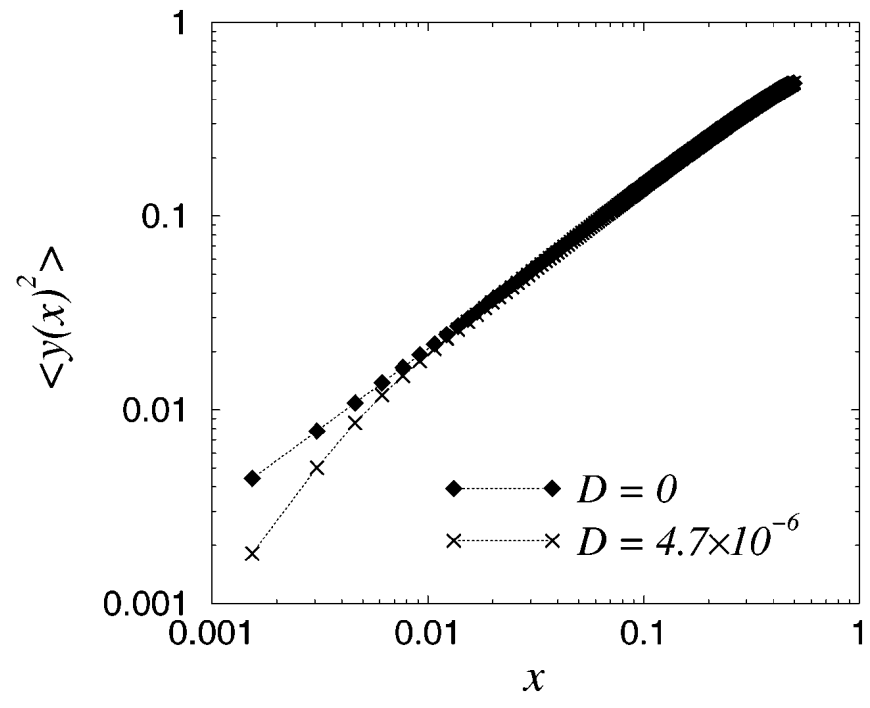

FIG. 4. Log-log plot of the second moment $\left\langle y(x)^{2}\right\rangle$ vs $x$ for the randomly forced FitzHugh-Nagumo Eqs. (6). Comparison between the cases with and without diffusion (i.e., $D=0$ and $\left.4.7 \times 10^{-6}\right)$ is made. The values of the other parameters are $a=1.0, b=0.55, \epsilon=0.1, K=5.0, \omega=0, \gamma=2.0$, and $f_{0}=5.0$.

length $2 \pi$ of the driving field which is much longer than $\sqrt{\epsilon D} \simeq 6.9 \times 10^{-4}$. Whether such a scaling regime with sufficient extension between two cutoff lengths is available in real systems (e.g., light-sensitive Belousov-Zhabotinsky reaction) will be a matter for a separate study.

One of the authors (D. B.) has been supported by The Monbusho's Grant-in-Aid for JSPS Postdoctoral Fellows.

*Present address: Physics and Technology Institute, Mongolian Academy of Sciences, Ulanbator 51, Mongolia.

[1] Y. Kuramoto, Chemical Oscillations, Waves, and Turbulence (Springer-Verlag, Berlin, 1984).

[2] M. C. Cross and P.C. Hohenberg, Rev. Mod. Phys. 65, 851 (1993).

[3] U. Frisch and G. Parisi, in Turbulence and Predictability in Geophysical Fluid Dynamics and Climate Dynamics, edited by M. Ghil, R. Benzi, and G. Parisi (North-Holland, Amsterdam, 1985).

[4] Y. Kuramoto, Prog. Theor. Phys. 94, 321 (1995); Int. J. Bifurcation Chaos Appl. Sci. Eng. 7, 789 (1997).

[5] Y. Kuramoto and H. Nakao, Phys. Rev. Lett. 76, 4352 (1996).

[6] Y. Kuramoto and H. Nakao, Phys. Rev. Lett. 78, 4039 (1997).

[7] R. Fitz-Hugh, Biophys. J. 1, 445 (1961); J. Nagumo, S. Arimoto, and S. Yoshizawa, Proc. IRE 50, 2061 (1962).

[8] G. Nicolis and I. Prigogine, Self-Organization in Nonequilibrium Systems, from Dissipative Structures to Order through Fluctuations (Wiley, New York, 1977).

[9] A.T. Winfree, The Geometry of Biological Time (Springer, New York, 1980).

[10] A.-L. Barabási and T. Vicsek, Phys. Rev. A 44, 2730 (1991); A.-L. Barabási, P. Szépfalusy, and T. Vicsek, Physica (Amsterdam) 178A, 17 (1991). 